\title{
Article \\ Nondestructive Tomographic Imaging of Rust with Rapid THz Time-Domain Spectroscopy
}

\author{
Hwan Sik Kim ${ }^{1}$, Seung Yeob Baik ${ }^{1}$, Joong Wook Lee ${ }^{2}$, Jangsun Kim ${ }^{3}\left[\right.$ and Yeong Hwan Ahn ${ }^{1, *}$ (i) \\ 1 Department of Physics and Department of Energy Systems Research, Ajou University, Suwon 16499, Korea; \\ ktj1214@naver.com (H.S.K.); rich194@ajou.ac.kr (S.Y.B.) \\ 2 Department of Physics and Optoelectronics Convergence Research Center, Chonnam National University, \\ Gwangju 61186, Korea; leejujc@chonnam.ac.kr \\ 3 Panoptics Corp., Seongnam 13516, Korea; jskim@panoptics.net \\ * Correspondence: ahny@ajou.ac.kr
}

check for updates

Citation: Kim, H.S.; Baik, S.Y.;

Lee, J.W.; Kim, J.; Ahn, Y.H.

Nondestructive Tomographic Imaging of Rust with Rapid $\mathrm{THz}$ Time-Domain Spectroscopy. Appl. Sci. 2021, 11, 10594. https://doi.org/ 10.3390/app112210594

Academic Editor: Mira Naftaly

Received: 18 October 2021

Accepted: 9 November 2021

Published: 11 November 2021

Publisher's Note: MDPI stays neutral with regard to jurisdictional claims in published maps and institutional affiliations.

Copyright: (c) 2021 by the authors. Licensee MDPI, Basel, Switzerland. This article is an open access article distributed under the terms and conditions of the Creative Commons Attribution (CC BY) license (https:// creativecommons.org/licenses/by/ $4.0 /)$.

\begin{abstract}
In this study, we developed a rapid nondestructive tool for testing rust spread in a metal covered by a paint layer by using a THz time-domain spectroscopy system at a speed of $100 \mathrm{~Hz} /$ pixel. Time-of-flight imaging helps identify rust formation by exclusively obtaining the reflection from the steel below the paint surface. The use of frequency-selective imaging allows us to manipulate the contrast in rust imaging. Higher contrast is generally obtained when monitoring using the higher frequency component. In addition, we monitored the spread of rust in a steel plate under the influence of two different chemical solutions: $\mathrm{NaCl}$ and acid. We found that in the early stages, the decrease in $\mathrm{THz}$ reflection was governed by the high-frequency components due to the formation of lepidocrocite, whereas the low-frequency component develops as the proportion of hematite increases with time.
\end{abstract}

Keywords: terahertz imaging; nondestructive testing; rust

\section{Introduction}

Rapid identification of rust in various metals is important to prevent structural failures in infrastructure, transportation, and utilities that use corrosive metals, as rust can lead to catastrophic failures [1-3]. In many cases, the metal is coated to provide stronger protection against corrosion. However, this metal is still vulnerable to corrosion as several paint ingredients are degradable, including additives [4-6]. In addition, the coating layers conceal the rust, which makes it difficult for the rust to be detected before structural failure becomes critical. Various nondestructive tests have been attempted for efficient and early detection of corrosion beneath the coating. However, their utility is limited when the metal surfaces are covered by a thick coating and are frequently obscured by lift-off effects [7-9]. For instance, ultrasonic testing cannot distinguish between the reflection from corrosion and that from other materials. Though the eddy current technique is a powerful tool for detecting corrosion, it can only be used to detect corrosion near the surface and is sensitive to other variations, except for conductance variation.

Recently, THz spectroscopy was introduced as an efficient nondestructive testing (NDT) tool for label-free sensing, material characterization, and inspection of the internal structures of objects [10-25]. In particular, time-domain spectroscopy (TDS) provides direct 3D mapping of an object by using the time-of-flight (TOF) of the reflected $\mathrm{THz}$ pulses. Conventional THz-TDS systems suffer from long acquisition times, and recently, novel THz-TDS systems based on compact femtosecond fiber laser technology have been introduced, delivering a rapid time delay of 100-500 Hz. For example, 3D imaging has been introduced with time-delay techniques such as electronically controlled optical sampling (ECOPS), asynchronous optical sampling (ASOPS), and optical sampling by cavity tuning (OSCAT) $[17,26,27]$. More recently, galvano-scanning systems have been incorporated 
into the rapid time-delay technique for the efficient inspection of defects in packaged chips, which provides information on the type of defects together with their locations [28]. Although $\mathrm{THz}$ imaging is a powerful tool for nondestructive mapping of conductive objects, the THz-TDS system has rarely been considered for NDT of rust embedded in the paint layer, particularly at imaging speeds higher than $100 \mathrm{~Hz} /$ pixel.

In this study, we report high-speed nondestructive imaging of rust on a metal plate covered by paint coatings. Using the THz-TDS system incorporated with galvano-mirror scanning, we obtained TOF imaging of the rust formation at a speed of $100 \mathrm{~Hz} / \mathrm{pixel}$. The $3 \mathrm{D}$ information was analyzed with respect to both the temporal and spectral domains. We also monitored the spread of rust when the steel plate was influenced by ionic and acidic solutions.

\section{Experimental Setup}

A schematic of the TOF imaging equipment is shown in Figure 1 Our rapid THz-TDS system is based on the commercialized OSCAT (TERA-OSCAT, MenloSystems GmbH, Germany), which allows for a sampling rate of 100-200 Hz/pixel; for instance, a measurement time of $16 \mathrm{~s}$ was allowed for each image with a size of $40 \times 40$ pixels at $100 \mathrm{~Hz} / \mathrm{pixel}$ [28]. The details of the experimental setup can be obtained from published literature, except that the $\mathrm{THz}$ imaging system was equipped with spring-loaded flexible arms, as shown in Figure 1. This made our THz-TOF system portable, enabling us to inspect metallic objects at various locations. Importantly, we incorporated a galvano-scanner to obtain the high-speed images with a scan rate that is limited by the OSCAT system. The THz signal was focused onto the rusted metal sample using a convex lens with a diameter of $3.8 \mathrm{~cm}$ and a focal length of $5 \mathrm{~cm}$. Conversely, the combined galvano-scanning ( $x$-axis) and slow stage scanning ( $y$-aixs) method can be used for larger samples (extending over $20 \mathrm{~mm}$ in one direction) and for inspecting the series of samples located in a row. The reflected $\mathrm{THz}$ signal was collected by the receiving antenna (denoted by Rx) using a beam splitter. Using home-built software, we recorded and analyzed 3D binary data as a function of the time delay, $x$-axis, and $y$-axis. We converted the time delay $(T)$ into depth information (i.e., in the $z$-axis), where 1 ps corresponds to $0.15 / n \mathrm{~mm}$ and $n$ is the refractive index of the material.

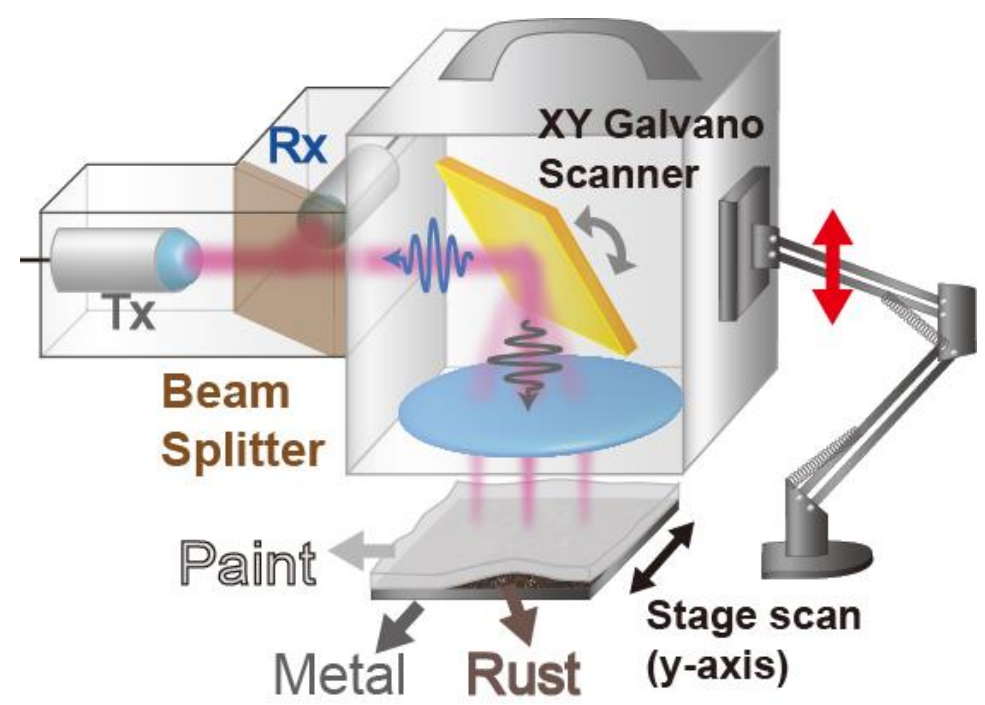

Figure 1. Schematic illustration of the THz-TOF imaging equipment. Tx and Rx denote the THz emitter and receiver, respectively, from the OSCAT THz-TDS system.

\section{Results and Discussion}

Figure $2 \mathrm{~b}$ shows the THz-TOF image of the rusted metal plate covered by a paint film, as shown in the photograph in Figure 2a. To verify the usefulness of our technique, we sprayed a paint layer with a thickness of $230 \mu \mathrm{m}$ on a partially rusted metal film. The inset 
of Figure 2a shows a photograph when we removed the paint film. Figure $2 b$ shows the time-integrated C-scan data (plotted as a function of the $x$ - and $y$-axes). The THz envelope signal obtained via the Hilbert transformation was time-integrated for each pixel. Here, the envelope function was obtained from the complex function, $\widetilde{E}_{\mathrm{THz}}=E_{\mathrm{THz}}+i H\left(E_{\mathrm{THz}}\right)$, where $E_{\mathrm{THz}}$ is the phase-sensitive $\mathrm{THz}$ amplitude from the measurement, and $H(x)$ is the Hilbert transformation of $x$ [29]. The scan range was $20 \times 30 \mathrm{~mm}^{2}$ with a pixel size of $80 \times 120 \mathrm{pixel}^{2}$. The bright signal in the upper middle region indicates the conductive metal region without rust formation, whereas the other regions are severely corroded. The formation of iron oxides, which strongly absorb the $\mathrm{THz}$ waves, is responsible for the reduced reflection in the rust region, and has been addressed elsewhere [30-32].
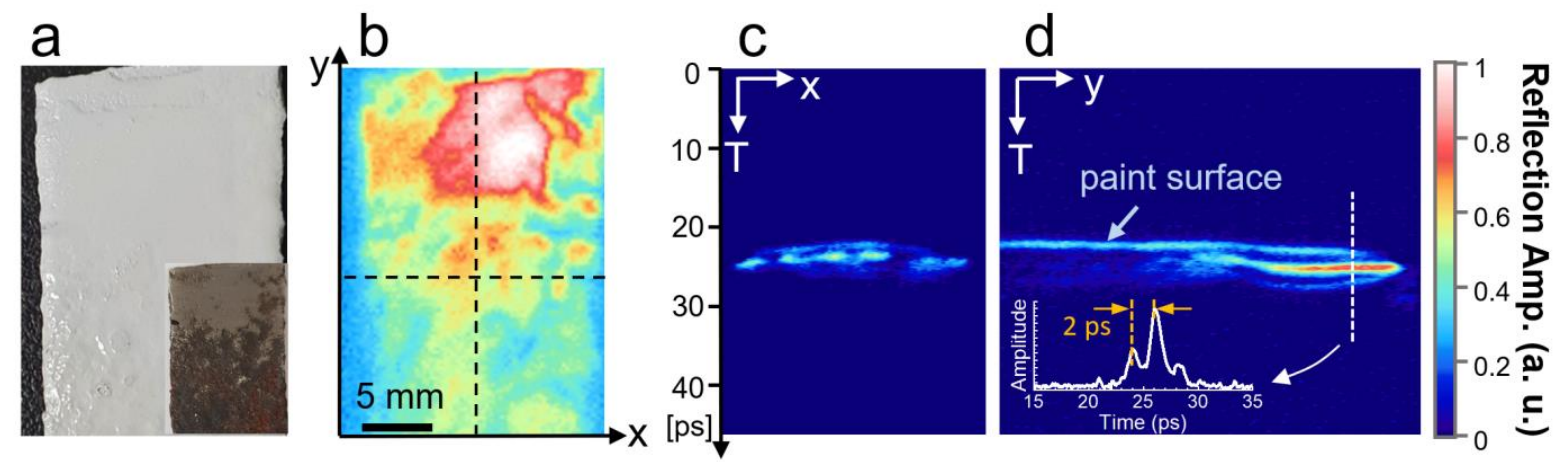

Figure 2. (a) Photograph of rusted metal concealed by paint. (b) Time-integrated C-scan image (in terms of envelope function) for (a). (c,d) Cross-sectional B-scan images along with the $x$-axis and $y$-axis taken from (b). (See dashed lines in (b)). Inset shows A-scan plot (i.e., THz amplitude vs. time-delay) obtained along the dashed line.

Two-dimensional tomographic images allow us to obtain depth profiles of the objects, reconstructed from the original 3D binary data. In other words, Figure $2 \mathrm{c}$ is a B-scan image in which the $\mathrm{THz}$ envelope signal is shown as a function of position $(x)$ along the horizontal dashed line in Figure 2b and time (T). Another B-scan image is shown in Figure 2d, which was taken along the vertical dashed line in Figure $2 \mathrm{~b}$. The rusted (where the reflected signal is weak) and the nonrusted regions can be clearly distinguished. The $\mathrm{THz}$ reflection at the paint's top surface and metal surface appeared at $T=24$ and $26 \mathrm{ps}$, respectively, as shown in the inset of Figure 2d. The TOF time difference of 2 ps between the top and bottom is consistent with the paint thickness of $230 \mu \mathrm{m}$, considering the refractive index of the paint $(n=2.6)$. The rusted area can be clearly identified in the TOF imaging, whereas the time-integrated imaging does not distinguish between the low reflection originating from the rust and that from the destructive interference. Therefore, THz-TOF imaging is advantageous over conventional NDT imaging techniques that are frequently obscured by the presence of lift-off in the paint layer [9].

Figure 3a shows a series of frequency-resolved C-scan images obtained using a Fourier transformation of the phase-sensitive $\mathrm{THz}$ amplitudes, which allows us to inspect the objects at specific spectral ranges. In other words, C-scan images were obtained for 0.4, 0.7, 1.0 , and $1.3 \mathrm{THz}$ (from left to right in the figure). As discussed earlier, the transverse resolution can be substantially improved by employing high-frequency components; in other words, the signal resolution improves from $1.8 \mathrm{~mm}$ at $0.4 \mathrm{THz}$ to $700 \mu \mathrm{m}$ at $1.0 \mathrm{THz}$ [28]. In addition to the improved spatial resolution, the reflection from the rust-rich region is suppressed more for the high-frequency components in general, whereas some of iron oxides have a relative strong absorption below $1 \mathrm{THz}$ [31]. Figure $3 \mathrm{~b}$ shows plots of the $\mathrm{THz}$ amplitude as a function of position along the vertical dashed line in Figure 3a, taken for different frequency components. THz reflection was suppressed relatively strongly at the rusted area (for $y>15 \mathrm{~mm}$ ) with increasing frequency. Therefore, it is desirable to have $\mathrm{THz}$ sources with higher frequency ranges for efficient nondestructive testing of the rusted metal with a better image contrast and transverse resolution. 


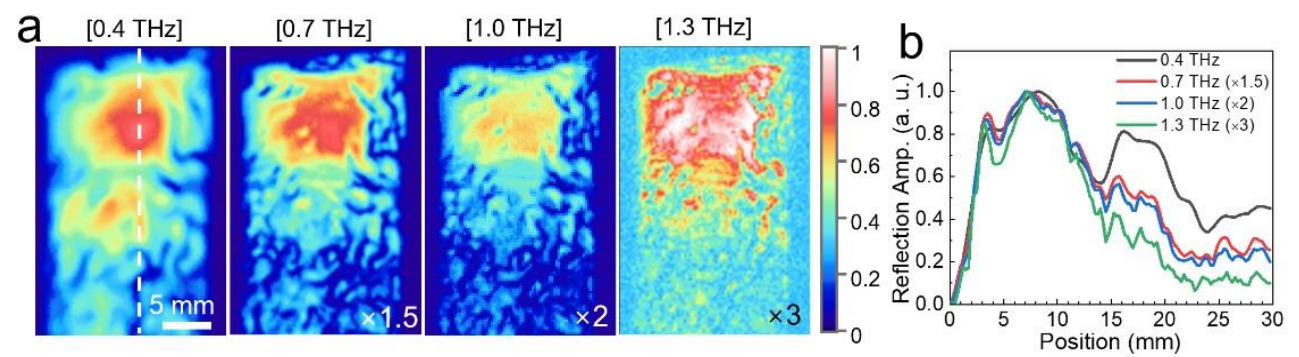

Figure 3. (a) A series of C-scan images corresponding to the frequencies of $0.4,0.7,1.0$, and $1.3 \mathrm{THz}$. Because the signal strength decreased toward the high-frequency region, the THz amplitude signal was magnified for clarity for the high-frequency images. (b) THz amplitudes as a function of position along the vertical dashed line in (a) for the different frequencies.

We monitored the spread of rust in a steel plate under the influence of two different ionic solutions. For instance, the use of $\mathrm{NaCl}$ solution induced rust in metal over a relatively long period of time. Conversely, acidic solutions (such as a mixture of hydrogen peroxide and acetic acid) resulted in a more rapid corrosion process. Figure 4a shows a series of photographs acquired over two weeks after the metal was submerged in $3.5 \% \mathrm{NaCl}$ solution. The corresponding $\mathrm{THz}$ images (i.e., time-integrated $\mathrm{C}$ images) are illustrated in Figure $4 \mathrm{~b}$. The $\mathrm{THz}$ reflection images were normalized by that of gold film. We temporarily extracted the sample from the solution for the measurements and returned it to the solution for further rust formation. Again, the scan range was $20 \times 30 \mathrm{~mm}^{2}$ with a pixel size of $80 \times 120$ pixel $^{2}$, and the THz signal was averaged five times per pixel this time. It was observed that the $\mathrm{THz}$ reflection was suppressed significantly with increasing time in the ionic solution. In a few regions, the $\mathrm{THz}$ amplitude reached less than $10 \%$ of the original amplitude (i.e., below $1 \%$ in terms of intensity).

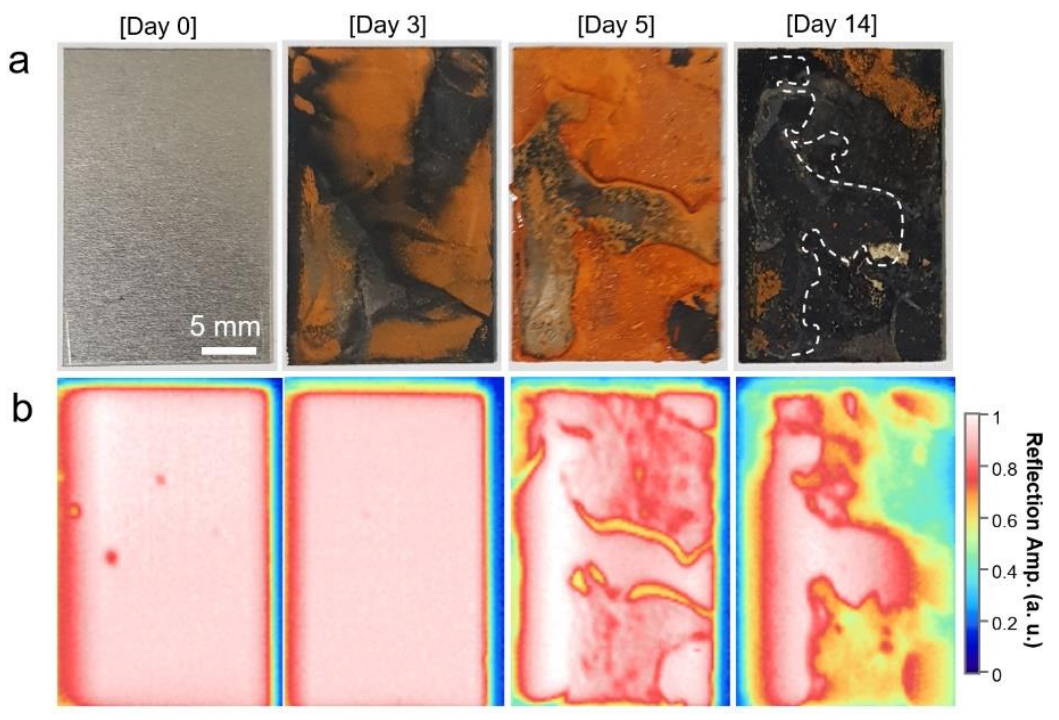

Figure 4. (a) Photographs of the steel plate taken on different days after the plate was submerged in the $\mathrm{NaCl}$ solution. (b) $\mathrm{THz}$ reflection amplitude images from the rusted metal plate taken on different days. Dashed line in (a) is a guide to eye for a direct comparison between the photographic image and $\mathrm{THz}$ image.

Importantly, the $\mathrm{THz}$ reflection images deviated significantly from the photographic images in most cases. For instance, the THz images taken on Day 3 showed a uniformly distributed reflection, which is similar to that of Day 0, whereas the photograph strongly implies rust formation over the entire surface. Similarly, in the dark region (surrounded by a dashed line) found in the photograph of Day 14, we found a bright reflection in the corresponding region of the $\mathrm{THz}$ reflection. In other words, the photograph was obscured 
by the shallow oxide layers (which tend to work as a protective layer to prevent further corrosion) and does not distinguish between the lightly and heavily rusted areas, which proves the usefulness of NDT with $\mathrm{THz}$ imaging techniques.

Figure 5 shows the results of rapid imaging where we applied an acidic solution (a mixture of $24 \mathrm{~mL}$ hydrogen peroxide (30\%), $1.1 \mathrm{~mL}$ acetic acid (13\%), and $4.5 \mathrm{~g} \mathrm{NaCl}$ ) to the steel plate. As the measurement time was $16 \mathrm{~s}$ for each image taken at $40 \times 40$ pixels, the rapid THz-TOF system was ideal for inspecting the rust spread that occurred over the course of a few minutes. Here, we dropped the acidic solution on the steel plate for fast rust formation, whereas we washed out the solution temporarily to take the $\mathrm{THz}$ imaging measurements each time. This is because we could not take the image in the presence of the solution and the bubbles that are produced violently in chemical reactions. We demonstrated a series of photographs and $\mathrm{THz}$ reflection images (i.e., time-integrated $\mathrm{C}$ images), respectively, in Figure 5a,b. Although the photographs did not show a noticeable change over time after the first $5 \mathrm{~min}$, the $\mathrm{THz}$ reflection images showed a continuous change, where the $\mathrm{THz}$ amplitude decreased to $80 \%$ of the original value. In other words, there was no strong correlation between the optical and THz reflection images, as shown in Figure 4.
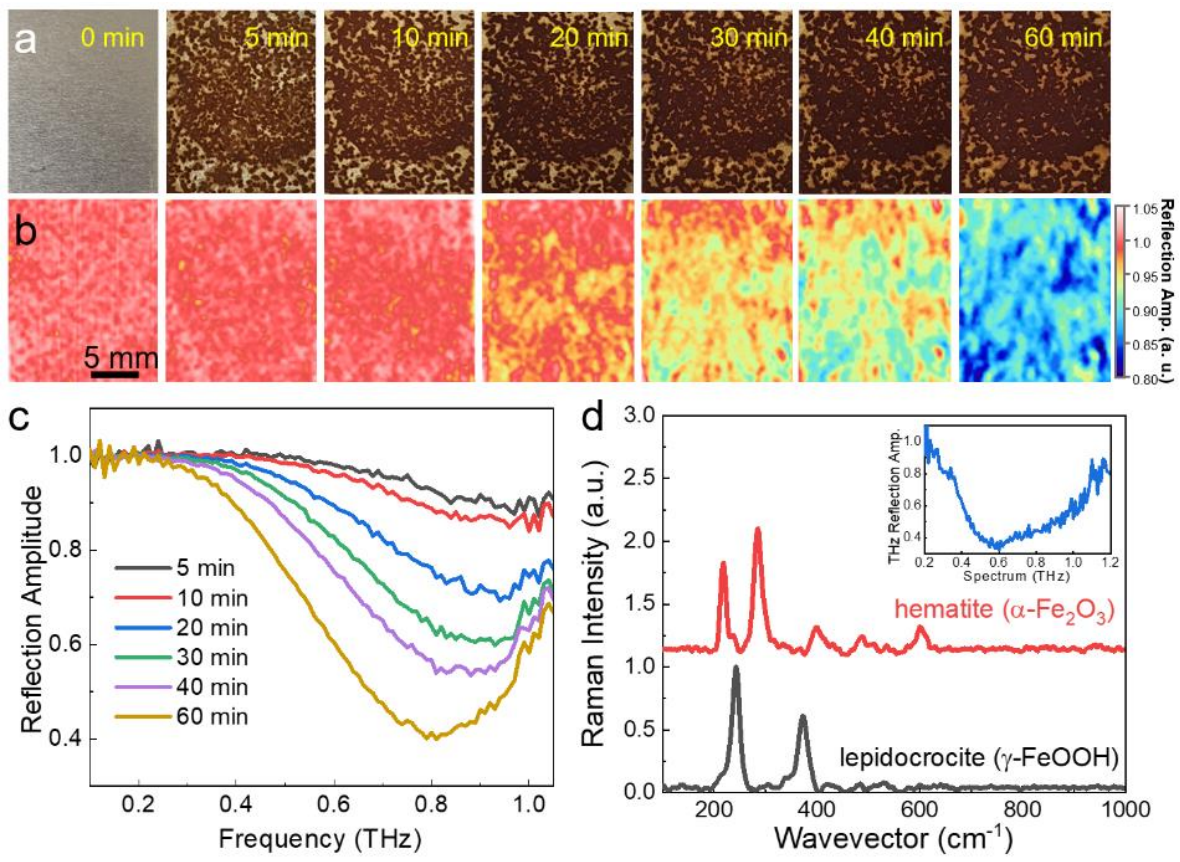

Figure 5. Photograph images (a) and rapid $\mathrm{THz}$ reflection images (b) for different measurement time after it is exposed to acidic solution. (c) THz reflection amplitude spectra averaged over the entire region in (b) for different measurement time. (d) Raman spectra of lepidocrocite and hematite found in our experiments. Inset shows the $\mathrm{THz}$ reflection signal from a thick hematite pallet.

Finally, in Figure $5 c$, we show the reflection spectra for different times averaged over the entire area in Figure 5b. We found that in the early stages, the decrease in the reflection (i.e., absorption by the iron oxides) was governed by the high-frequency component, whereas the lower-frequency component becomes more pronounced with time. In the end, the absorption peaked at approximately $0.8 \mathrm{THz}$ on average after $60 \mathrm{~min}$. Interestingly, we observed dynamic transitions between the different oxide types. However, their dynamic behavior in terms of different types of metal oxides has not been addressed previously. We observed a very similar tendency for the data shown in Figure 4 with the slow corrosion processes, in which the absorption peak reached $0.8 \mathrm{THz}$ after Day 14 (when the reflection amplitude reached less than $10 \%$ of the initial value).

To unravel the oxide types responsible for the $\mathrm{THz}$ reflection change, we performed Raman spectroscopy, as shown in Figure 5d. We used a laser source at $632.8 \mathrm{~nm}$, focused 
with a spot size of $1 \mu \mathrm{m}$. We identified two types of iron oxides from the rusted area; i.e., lepidocrocite $(\gamma-\mathrm{FeOOH})$ and hematite $\left(\alpha-\mathrm{Fe}_{2} \mathrm{O}_{3}\right)$. It is likely that the initial frequency response is governed by $\gamma-\mathrm{FeOOH}$ whose $\mathrm{THz}$ absorption is known to be pronounced in the higher frequency range [33]. Contrarily, the THz response of $\alpha-\mathrm{Fe}_{2} \mathrm{O}_{3}$ has not been reported; we performed $\mathrm{THz}$ reflection measurement on a thick $\alpha-\mathrm{Fe}_{2} \mathrm{O}_{3}$ pallet, which showed a strong absorption at around $0.6-0.8 \mathrm{THz}$ (inset of Figure $5 \mathrm{~d}$ ). Therefore, we conclude that the transition toward the lower frequency region over time was mainly due to the increased amount of $\alpha-\mathrm{Fe}_{2} \mathrm{O}_{3}$. The monitoring of dynamical behavior in chemical reactions is crucial for optimizing material processing. Therefore, our study necessitates future investigation on the development of in situ $\mathrm{THz}$ imaging tools, for instance, by incorporating fluidic devices that enable the use of thin chemical solutions for efficient $\mathrm{THz}$ detection.

\section{Conclusions}

In this study, we conducted rapid nondestructive testing on rusted metal using a highspeed THz-TDS spectroscopy system at a rate of $100 \mathrm{~Hz} /$ pixel. The rusted area was clearly identified in the TOF imaging in the presence of the paint layer, which is advantageous over conventional NDT imaging techniques that are frequently obscured when there is lift-off in the protective layer. In addition, the $\mathrm{THz}$ reflection images enable us to distinguish between the lightly and heavily rusted area in contrast to the photographic images. Via Fourier transformation of the phase-sensitive signals, we obtained frequency-selective imaging in the range 0.2-1.3 THz. Overall, a higher contrast was achieved with the higher frequency component. In addition, we monitored the spread of rust in a steel plate when the steel was submerged in $\mathrm{NaCl}$ and acidic solution (a mixture of hydrogen peroxide and acetic acid). We found that in the early stages, the decrease in the $\mathrm{THz}$ reflection signal was governed by the high-frequency components, whereas the contrast in the lower frequency range developed for longer periods. In other words, the absorption peak reached $0.8 \mathrm{THz}$ as hematite was formed over a long period of time. $\mathrm{THz}$ response on a ferrous oxide pallet (combined with the Raman spectroscopy results) revealed the dynamic transition between the different oxide types, i.e., from lepidocrocite to hematite. Consequently, the rapid imaging technique was found to be a very powerful tool for the nondestructive inspection of rust in metals, including their dynamic behaviors.

Author Contributions: Conceptualization, Y.H.A.; formal analysis, H.S.K., S.Y.B. and Y.H.A.; investigation, H.S.K. and S.Y.B., J.W.L. and Y.H.A.; resources, J.K.; writing-original draft, H.S.K. and Y.H.A.; writing-review and editing, H.S.K., J.W.L., J.K. and Y.H.A. All authors have read and agreed to the published version of the manuscript.

Funding: This work was supported by the Midcareer Researcher Program (2020R1A2C1005735) and Basic Science Research Program (2021R1A6A1A10044950) through a National Research Foundation grant funded by the Korea Government, and by the Human Resources Program in Energy Technology (20184030202220) of the Korea Institute of Energy Technology Evaluation and Planning (KETEP) grant funded by the Korea Government.

Institutional Review Board Statement: Not applicable.

Informed Consent Statement: Not applicable.

Data Availability Statement: Data are contained within the article.

Conflicts of Interest: The authors declare no conflict of interest.

\section{References}

1. Shaw, B.; Kelly, R. What is corrosion? Electrochem. Soc. Interface 2006, 15, 24. [CrossRef]

2. El-Meligi, A. Corrosion Preventive Strategies as a Crucial Need for Decreasing Environmental Pollution and Saving Economics. Re. Pat. Corr. Sci. 2010, 2, 22-33. [CrossRef]

3. Hansson, C.M. The impact of corrosion on society. Metall. Mater. Trans. 2011, 42, 2952-2962. [CrossRef]

4. Leidheiser, H., Jr. Corrosion of Painted Metals A Review. Corrosion 1982, 38, 374-383. [CrossRef] 
5. Morcillo, M. Soluble salts: Their effect on premature degradation of anticorrosive paints. Prog. Org. Coat. 1999, 36, 137-147. [CrossRef]

6. Chico, B.; Morcillo, M. The effects of soluble salts at the metal/paint interface: Advances in knowledge. Port. Electrochim. Acta 2006, 24, 191-206.

7. Rifai, D.; Abdalla, A.N.; Ali, K.; Razali, R. Giant magnetoresistance sensors: A review on structures and non-destructive eddy current testing applications. Sensors 2016, 16, 298. [CrossRef]

8. Si, D.; Gao, B.; Guo, W.; Yan, Y.; Tian, G.Y.; Yin, Y. Variational mode decomposition linked wavelet method for EMAT denoise with large lift-off effect. NDT E Int. 2019, 107, 102149. [CrossRef]

9. Wu, R.; Zhang, H.; Yang, R.; Chen, W.; Chen, G. Nondestructive Testing for Corrosion Evaluation of Metal under Coating. J. Sens. 2021, 2021. [CrossRef]

10. Kawase, K.; Ogawa, Y.; Watanabe, Y.; Inoue, H. Non-destructive terahertz imaging of illicit drugs using spectral fingerprints. Opt. Express 2003, 11, 2549-2554. [CrossRef]

11. Federici, J.F.; Schulkin, B.; Huang, F.; Gary, D.; Barat, R.; Oliveira, F.; Zimdars, D. THz imaging and sensing for security applications-Explosives, weapons and drugs. Semicond. Sci. Technol. 2005, 20, S266-S280. [CrossRef]

12. Shen, Y.C.; Lo, T.; Taday, P.F.; Cole, B.E.; Tribe, W.R.; Kemp, M.C. Detection and identification of explosives using terahertz pulsed spectroscopic imaging. Appl. Phys. Lett. 2005, 86, 1-3. [CrossRef]

13. Zhong, H.; Xu, J.; Xie, X.; Yuan, T.; Reightler, R.; Madaras, E.; Zhang, X.C. Nondestructive defect identification with terahertz time-of-flight tomography. IEEE Sens. J. 2005, 5, 203-207. [CrossRef]

14. Karpowicz, N.; Redo, A.; Zhong, H.; Li, X.; Xu, J.; Zhang, X.C. Continuous-wave terahertz imaging for non-destructive testing applications. In Proceedings of the Joint 30th International Conference on Infrared and Millimeter Waves and 13th International Conference on Terahertz Electronics, Williamsburg, VA, USA, 19-23 September 2005.

15. Schirmer, M.; Fujio, M.; Minami, M.; Miura, J.; Araki, T.; Yasui, T. Biomedical applications of a real-time terahertz color scanner. Biomed. Opt. Express 2010, 1, 354-366. [CrossRef]

16. Kawase, K.; Shibuya, T.; Hayashi, S.; Suizu, K. THz imaging techniques for nondestructive inspections. Comptes Rendus Phys. 2010, 11, 510-518. [CrossRef]

17. Jin, K.H.; Kim, Y.-G.; Cho, S.H.; Ye, J.C.; Yee, D.-S. High-speed terahertz reflection three-dimensional imaging for nondestructive evaluation. Opt. Express 2012, 20, 25432-25440. [CrossRef] [PubMed]

18. Park, S.H.; Jang, J.W.; Kim, H.S. Non-destructive evaluation of the hidden voids in integrated circuit packages using terahertz time-domain spectroscopy. J. Micromech. Microeng. 2015, 25, 095007. [CrossRef]

19. Park, S.J.; Yoon, S.A.N.; Ahn, Y.H. Dielectric constant measurements of thin films and liquids using terahertz metamaterials. RSC Adv. 2016, 6, 69381-69386. [CrossRef]

20. Fan, S.; Li, T.; Zhou, J.; Liu, X.; Liu, X.; Qi, H.; Mu, Z. Terahertz non-destructive imaging of cracks and cracking in structures of cement-based materials. AIP Adv. 2017, 7, 115202. [CrossRef]

21. Park, S.J.; Kim, A.R.; Hong, J.T.; Park, J.Y.; Lee, S.; Ahn, Y.H. Crystallization Kinetics of Lead Halide Perovskite Film Monitored by In Situ Terahertz Spectroscopy. J. Phys. Chem. 2017, 8, 401-406. [CrossRef] [PubMed]

22. Kim, H.S.; Sung, H.C.; Roy, B.; Kim, S.; Ahn, Y.H. Humidity sensing using THz metamaterial with silk protein fibroin. Opt. Express 2018, 26, 33575-33581. [CrossRef]

23. Ahi, K.; Shahbazmohamadi, S.; Asadizanjani, N. Quality control and authentication of packaged integrated circuits using enhanced-spatial-resolution terahertz time-domain spectroscopy and imaging. Opt. Lasers Eng. 2018, 104, 274-284. [CrossRef]

24. Zhang, J.Y.; Ren, J.J.; Li, L.J.; Gu, J.; Zhang, D.D. THz imaging technique for nondestructive analysis of debonding defects in ceramic matrix composites based on multiple echoes and feature fusion. Opt. Express 2020, 28, 19901-19915. [CrossRef] [PubMed]

25. Kim, H.S.; Ha, N.Y.; Park, J.Y.; Lee, S.; Kim, D.S.; Ahn, Y.H. Phonon-Polaritons in Lead Halide Perovskite Film Hybridized with THz Metamaterials. Nano Lett. 2020, 20, 6690-6696. [CrossRef] [PubMed]

26. Bartels, A.; Cerna, R.; Kistner, C.; Thoma, A.; Hudert, F.; Janke, C.; Dekorsy, T. Ultrafast time-domain spectroscopy based on high-speed asynchronous optical sampling. Rev. Sci. Instrum. 2007, 78, 035107. [CrossRef]

27. Wilk, R.; Hochrein, T.; Koch, M.; Mei, M.; Holzwarth, R. OSCAT: Novel technique for time-resolved experiments without moveable optical delay lines. J. Infrared Millim. Terahertz Waves 2011, 32, 596-602. [CrossRef]

28. Yim, J.H.; Kim, S.Y.; Kim, Y.; Cho, S.; Kim, J.; Ahn, Y.H. Rapid 3d-imaging of semiconductor chips using thz time-of-flight technique. Appl. Sci. 2021, 11, 4770. [CrossRef]

29. Kong, D.Y.; Wu, X.J.; Wang, B.; Gao, Y.; Dai, J.; Wang, L.; Ruan, C.J.; Miao, J.G. High resolution continuous wave terahertz spectroscopy on solid-state samples with coherent detection. Opt. Express 2018, 26, 17964-17976. [CrossRef]

30. Komatsu, M.; Sato, R.; Mizuno, M.; Fukunaga, K.; Ohki, Y. Feasibility study on terahertz imaging of corrosion on a cable metal shield. Jpn. J. Appl. Phys. 2012, 51, 122405. [CrossRef]

31. Wu, D.B.; Zhao, H.; Liu, H.L.; Zhao, K.; Sun, Q. THz spectrum of iron corrosion products. In Proceedings of the Selected Photoelectronic Technology Committee Conferences, China, China, 1 August 2014.

32. Fuse, N.; Sugae, K. Non-destructive terahertz imaging of alkali products in coated steels with cathodic disbanding. Prog. Org. Coat. 2019, 137, 105334. [CrossRef]

33. Fuse, N.; Fukuchi, T.; Takahashi, T.; Mizuno, M.; Fukunaga, K. Evaluation of applicability of noncontact analysis methods todetect rust regions in coated steel plates. IEEE Trans. Terahertz Sci. Technol. 2012, 2, 242-249. [CrossRef] 